- clarify the need for the update

- scope the evidence base and define the breadth of the remit

- follow established processes and work within existing methodology

- manage guideline development group expectations.

\section{P008 KEEPING CANCER GUIDELINES CURRENT USING A WIKI APPROACH}

Ian Olver (Australia)

10:1136/bmjqs-2013-002293.8

Updating written guidelines regularly is difficult and expensive. New evidence in cancer treatment is published frequently. Guideline booklets are also difficult to disseminate widely and stakeholder feedback is mainly pre-publication. To address these issues, Cancer Council Australia developed a web-based wiki platform for guidelines. Only invited expert authors can write in the wiki guidelines, but any stakeholder can comment upon them at any time. The key steps in guideline development were integrated with the wiki capability. An expert group, whose competing interests are documented, were identified, the key clinical questions and search strategies were developed for each question and literature searches recorded on the wiki. An online literature screening and critical appraisal process was developed. Evidencebased recommendations were formulated and evidence tables automatically generated. The stakeholders were invited to comment online. Web analytics monitored usage. The writers remain engaged to appraise new papers and update the guideline rapidly as necessary. All previous versions could be accessed. We evaluated lung cancer treatment guidelines developed on the wiki, where 22 authors identified 67 clinical questions. The literature search and screening process resulted in 2035 potentially relevant articles being forwarded for detailed methodological evaluation with another 571 added through snowballing and other methods. To fine-tune the initial draft content, the working party used the wiki to exchange 156 internal comments in 9 weeks. Of 1055 visits in a 30 day initial consultation period 487 were targeted by email and 387 found the site by Google searches. Of respondents from 45 countries, most were from Australia (799 visits), New Zealand (60 visits) and the United States (31 visits) Of 38 comments, 31 resulted in edits. A strategy to boost uptake is to write Qstream education modules to accompany the guidelines.

\section{Plenary 4: Developing Implementable Guidelines}

\section{P009 GUIDELINE IMPLEMENTABILITY: LEARNING FROM GREAT THINKERS LIKE PICASSO, THE DALAI LAMA AND ANONYMOUS}

Melissa Brouwers (Canada)

\section{0:1136/bmjqs-2013-002293.9}

A tension faced in the guideline enterprise is finding the right balance between development, implementation and evaluation of recommendations. Are developers getting mired in the minutiae of creating the best guideline report? Are implementers choosing the best messages and strategies to optimise utilisation? Are evaluators assessing the most meaningful outcomes or the outcomes that are easiest to measure? Together, are these players in the guideline enterprise optimising what is known in their respective fields and the relationships among each other to create a system for success? In this presentation, we will turn for inspiration to some great thinkers, consider the research environment, and bring in real life examples to address these questions.

\section{P010 DEVELOPING AND EVALUATING COMMUNICATION STRATEGIES TO SUPPORT INFORMED DECISIONS AND PRACTICE BASED ON EVIDENCE (DECIDE) FOR HEALTH PROFESSIONALS}

Pablo Alonso (Spain).

10:1136/bmjqs-2013-002293.10

The DECIDE initiative, building on the work of GRADE, is exploring methods to ensure effective communication of evidence-based recommendations targeted at key stakeholders (healthcare professionals, policymakers, and managers, and patients and the general public). DECIDE will produce strategies for communicating recommendations that are being rigorously evaluated in diverse settings, and it will support the transfer of research into practice in healthcare systems globally. The methodology is an iterative process that includes; brainstorming, user feedback through user testing, surveys, trials and implementation and evaluation in real guidelines. All this is being done across a wide range of healthcare systems in Europe, North America, and other countries.

The work with healthcare professionals is developing three strategies: 1) An electronic multilayered guideline format that presents the essential information that healthcare professionals tell us they need to understand and act on a recommendation; 2) An evidence to recommendation table for users: this is a summary table with the factors for moving from evidence to a recommendation and the justification for each recommendation; 3) A decision aid template to semi-automatically build decision aids linked to guidelines to be used at the point of care. For strategies 1 and 3 DECIDE is also collaborating with the MAGIC programme.

These strategies will be implemented a guideline authoring tool that is being developed from GRADEpro (called the Guideline Development Tool, or GDT), and will also be implementable in other online guideline authoring tools, allowing guideline groups to decide which of these strategies to use when developing their guidelines. The GDT will be able to facilitate the full authoring of a typical guideline and allow the updating of these strategies when needed. Guideline outputs will be made available through multiple platforms (e.g., websites, smartphones and tablets apps).

These tools will help to make guidelines better suited to the information needs of health professionals, policymakers and consumers across diverse healthcare settings in Europe and elsewhere.

\section{P011 SUCCESS AND CHALLENGES FROM OVER 5 YEARS OF THE NATIONAL STROKE FOUNDATION'S STROKELINK PROGRAM. AN EXAMPLE OF A COMPREHENSIVE IMPLEMENTATION PROGRAM LINKING STROKE GUIDELINES TO CURRENT PRACTICE IN AUSTRALIA}

Kelvin Hill (Australia).

10:1136/bmjqs-2013-002293.11 